\title{
Auditory Neuropathy/Dyssynchrony: A Retrospective Analysis of 15 Cases
}

\author{
Murat Unal $^{1} \quad$ Yusuf Vayisoglu $^{1}$ \\ ${ }^{1}$ Department of Otorhinolaryngology, Mersin University, \\ Mersin, Turkey

\begin{abstract}
Address for correspondence Yusuf Vayisoglu, MD, Department of Otorhinolaryngology, Mersin University, Mersin 33079, Turkey (e-mail: yvayisoglu@gmail.com).
\end{abstract}

Int Arch Otorhinolaryngol 2015;19:151-155.

Abstract
Keywords
- auditory neuropathy/
dyssynchrony
- otoacoustic emission
- auditory brainstem
response

Introduction Auditory neuropathy/dyssynchrony (AN/AD) comprises a spectrum of pathology affecting the auditory pathways anywhere from the inner hair cells to the brainstem. It is characterized by an absent or atypical auditory brainstem response (ABR) with preservation of the cochlear microphonics and/or otoacoustic emissions (OAEs).

Objective Retrospective analysis of patients with AN/AD.

Methods Fifteen patients with AN/AD were included in this study and their records were retrospectively investigated.

Results Possible etiology of AN/AD was neonatal hyperbilirubinemia in three patients, family history of hearing loss in three patients, consanguineous marriage in two patients, head trauma in two patients, mental motor retardation in one patient, cerebrovascular disease in one patient, and there was no apparent cause in three patients.

Conclusion Otolaryngologists should keep in mind the diagnosis of AN/AD especially in patients complaining of difficulty in hearing and speech and audiological evidence of disassociation between pure tone and speech audiometry. ABR and OAE testing is recommended in these patients for AN/AD diagnosis.

\section{Introduction}

Auditory neuropathy/dyssynchrony (AN/AD) is a hearing disorder characterized by an absent or atypical auditory brainstem response (ABR), with preservation of the cochlear microphonics (CM) and/or otoacoustic emissions (OAEs). ${ }^{1,2}$ In 1996, Starr et al first described this rare entity, drawing on their observations in 10 patients. ${ }^{3}$ The authors suggested that these patients were probably similar to those previously reported cases with a paradoxical absence of ABRs and only a slight impairment of pure tone thresholds but in whom CMs or OAEs had not been recorded. ${ }^{3-5}$ Also In 1998, Doyle et al reported eight patients with normal transient evoked OAEs (TEOAEs) and distortion product OAEs (DPOAEs) combined with the absence or marked abnormalities of ABRs. ${ }^{6}$ Starr et al suggested that this type of hearing impairment is due to a disorder that impairs auditory nerve function and may have as one of its causes a neuropathy of the auditory nerve, occurring either in isolation or as part of a systemic neuropathic process. ${ }^{3}$ Clinically, the diagnostic criteria of AN/AD is defined as (1) sensorineural hearing loss, usually bilateral, of any degree; (2) normal outer hair cell function as evidenced by the presence of OAEs and/or CM; (3) absent or atypical ABR; (4) understanding of speech worse than would be predicted from the behavioral or pure tone audiometry; (5) absent acoustic reflexes to the ipsilateral and contralateral tones a $110-\mathrm{dB}$ hearing level. ${ }^{1,7-9}$

In this retrospective study, we investigated the audiological findings, history, and clinical manifestations of patients diagnosed with $\mathrm{AN} / \mathrm{AD}$ at our clinic. received

June 4, 2014

accepted after revision

August 25, 2014

published online

November 28, 2014
DOI http://dx.doi.org/

10.1055/s-0034-1390328. ISSN 1809-9777.
Copyright $\odot 2015$ by Thieme Publicações License terms Ltda, Rio de Janeiro, Brazil 


\section{Patients and Methods}

Fifteen patients with AN/AD were included in this study, and their records were retrospectively investigated. All of the patients' medical and otologic histories were recorded. A complete ear, nose, and throat examination was performed. Otoscopic examinations were done by an otolaryngologist before testing to rule out any external or middle ear pathology that could affect audiometric measurements. Then pure tone audiometry (250 to $8,000 \mathrm{~Hz}$ ), tympanometry, and acoustic reflex measurement ( 500 to $4,000 \mathrm{~Hz}$ ) were done in a standard fashion (Interacoustic AC 40 and AZ 26, Denmark, Assens). DPOAEs were measured at click levels of 65 (L1) and $55 \mathrm{~dB}$ (L2) peak sound pressure for the F1 and F2 components (Homoth Medizinelektronik GmbH\&Co, KG, Germany, Hamburg). DPOAE-grams were recorded in onequarter-octave steps over a frequency range of F2 from 0.5 to $6 \mathrm{kHz}$. DPOAE values were plotted on a DPOAEgram, which shows the emission level as a function of the F2 frequency. ABRs were recorded using Homoth ABR equipment. Electrodes were placed on the forehead as a ground electrode and on both mastoids as active electrodes. The responses were filtered with a bandpass of 100 to $3,000 \mathrm{~Hz}$. Alternate polarity clicks of 100-millisecond duration were presented monaurally with a repetition rate of $16.4 / \mathrm{s}$. Each ear was tested separately, and all responses were replicated twice. Patients underwent assessment in a state of natural sleep. In this retrospective study, we did not include genetic research; we investigated only according to the history of patients.

\section{Results}

Eleven male and four female patients diagnosed with AN/AD (age range: 2 to 52 years and median age: 19.3 years) were included in this study. Possible etiology of AN/AD was neonatal hyperbilirubinemia in three patients. These patients had a history of exchange transfusion because of bilirubin levels over $20 \mathrm{mg} / \mathrm{dL}$. Three patients had family history of hearing loss, two patients had consanguineous marriage, two patients had head trauma, one patient had mental motor retardation (psychomotor retardation), one patient had cerebrovascular disease, and there was no apparent causes in three patients.

Thirteen patients had bilateral and two patients had unilateral AN/AD.

None of the patients with AN/AD had middle or inner ear anomalies on computed tomography or magnetic resonance imaging. - Table 1 summarizes the patients' demographic, clinical, and audiological features (see also - Fig. 1 for a left and right ear ABR and DPOAE recording of a case of AN/AD).

Eight of 15 patients' pure tone audiometric results showed profound hearing losses; however, seven of them had mild to moderate hearing loss. Acoustic reflex tests were also absent in all of the patients.

\section{Discussion}

AN/AD is characterized by a unique pattern of hearing loss and distorted $A B R$ with preservation of outer hair cell function. ${ }^{10,11} \mathrm{AN} / \mathrm{AD}$ comprises a spectrum of pathology affecting the auditory pathways anywhere from the inner hair cells to the brainstem. Thus it is difficult to define the disorder as cochlear or retrocochlear. Increased clinical suspicion supported by appropriate diagnostic tests is needed to establish an accurate diagnosis. ${ }^{12,13}$

The clinical findings for auditory neuropathy are associated with several diagnoses including hyperbilirubinemia, neurodegenerative diseases, Charcot-Marie-Tooth syndrome, and other sensorimotor neuropathologies, mitochondrial

Table 1 Clinical and audiological features of the patients with AN/AD

\begin{tabular}{|l|l|l|l|l|l|}
\hline Patient No. & Age $(\mathrm{y})$ & Sex & ABR & DPOAE & History \\
\hline 1 & 2 & M & Bilateral absent & Bilateral present & Consanguineous marriage \\
\hline 2 & $3 y$ & M & Bilateral absent & Bilateral present & Mental motor retardation \\
\hline 3 & 52 & F & Bilateral absent & Right ear $(+)$ & Trauma \\
\hline 4 & 13 & F & Bilateral absent & Bilateral present & Blood exchange due to hyperbilirubinemia \\
\hline 5 & 2 & M & Bilateral absent & Right ear $(+)$ & No apparent cause \\
\hline 6 & 2 & F & Bilateral absent & Bilateral present & Blood exchange due to hyperbilirubinemia \\
\hline 7 & 7 & F & Bilateral absent & Bilateral present & Family history of hearing loss \\
\hline 8 & 4 & M & Bilateral absent & Bilateral present & No apparent cause \\
\hline 9 & 30 & M & Bilateral absent & Bilateral present & Trauma \\
\hline 10 & 5 & M & Bilateral absent & Bilateral present & Consanguineous marriage \\
\hline 11 & 2 & M & Bilateral absent & Bilateral present & Blood exchange due to hyperbilirubinemia \\
\hline 12 & 52 & M & Bilateral absent & Bilateral present & Family history of hearing loss \\
\hline 13 & 29 & M & Bilateral absent & Bilateral present & No apparent cause \\
\hline 14 & 6 & M & Bilateral absent & Bilateral present & Family history of hearing loss \\
\hline 15 & 50 & M & Bilateral absent & Bilateral present & Cerebrovascular disease \\
\hline
\end{tabular}

Abbreviations: ABR, auditory brain stem response; AN/AD, auditory neuropathy/dyssynchrony; DPOAE, distortion product otoacoustic emission. 
RIGHT (Imp = 2485 Ohm)
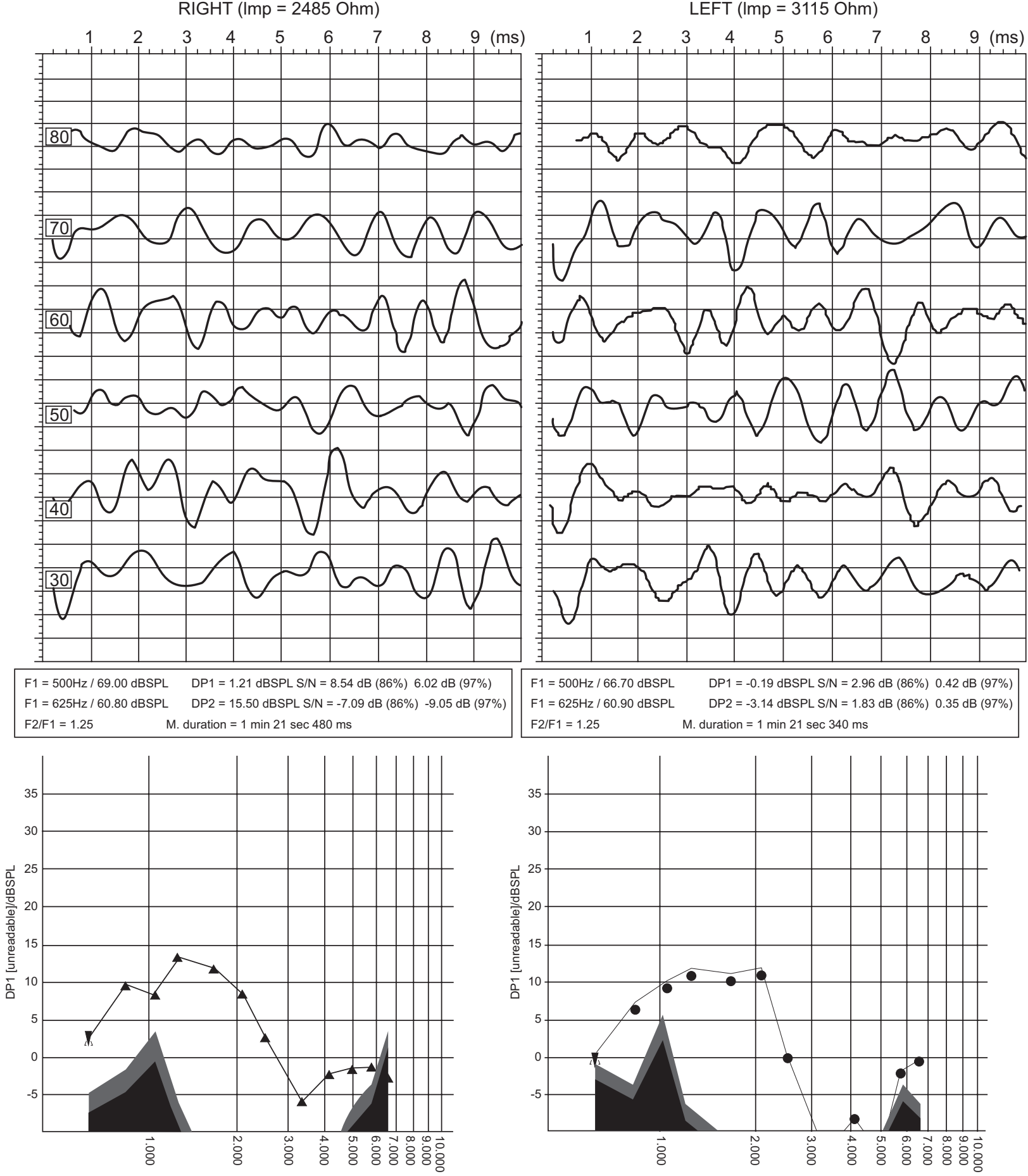

Fig. 1 Left and right ear auditory brain stem response and distortion product otoacoustic emission recording of a case of auditory neuropathy/ dyssynchrony. Abbreviations: dBSPL, decibels sound pressure level; DP, distortion product; F1 and F2 used as formulation markers.

disorders, and ischemic-hypoxic neuropathy resulting from asphyxia. ${ }^{14}$ Also, experimental animal models for auditory neuropathy have been proposed using the carboplatin ototoxicity and ischemic-hypoxic neuropathy methodologies. $^{12,15,16}$ In our series, three patients with AN/AD had neonatal hyperbilirubinemia, three patients had family history of hearing loss, two patients had consanguineous marriage, two patients had head trauma, one patient had mental motor retardation, and one patient had cerebrovascular disease. We did not find any specific etiology in three patients.

Recently two mechanisms have been proposed for explaining the abnormalities of auditory function: (1) impaired synchrony among nerve fibers and/or (2) reduced neural input. ${ }^{17-19}$ It is generally thought that absent or severely distorted ABR is highly likely to be related to impairment of 
neural synchrony in the auditory pathways. Similar to the previous reports, we could not obtain any response to the click stimulus.

The prevalence of AN/AD in patients with hearing loss ranged from 0.5 to $15 \%$ according to studies in literature. ${ }^{10,11}$ Because there are so many possible causes for AN/AD, it is difficult to estimate its exact prevalence. ${ }^{14}$ Davis and Hirsh suggested that 1 in every 200 hearing-impaired children had ABR findings inconsistent with pure tone findings. ${ }^{20}$ Permanent bilateral hearing loss is seen in 1.4 per 1,000 live births. Therefore the incidence of AN/AD is likely to be 1.4 per 10,000 live births. According to Rance et al, AN/AD would be present in 2.3 per 1,000 infants with risk factors for hearing loss. Thus, if OAE tests alone are used for hearing screening of infants with risk factors, $11 \%$ of infants with permanent hearing loss will be missed. ${ }^{14,21}$ These data are important for designing the newborn hearing screening protocols especially in developing countries.

Patients with AN/AD complain of hearing disability, especially in the presence of noise, and tend to have wordrecognition scores that are disproportionately poorer than would be expected by audiometric thresholds. In our series, all of the patients were admitted to our department with the complaint of hearing and speech disability. Only one of them had understandable speech. Pure tone audiometric thresholds are variable in patients with AN/AD, and the degree of hearing loss can range from mild to profound sensorineural type. Eight of our patients' pure tone audiometric results showed profound hearing losses, and seven had mild to moderate hearing loss. Acoustic reflex tests were also absent in all of the patients. Because OAEs and CM are dependent on the integrity of cochlear outer hair cells and are "preneural" events, they may be present and normal in AN/AD; however, absent or grossly abnormal ABRs are seen. ${ }^{14,22}$ Similar findings were observed in our cases.

The hallmark of AN/AD is an abnormal ABR reading together with a normal OAE reading. However, there is a lack of actual diagnostic procedures for AN/AD. Other tests may also be used as part of a more comprehensive evaluation of an individual's hearing and speech-perception abilities. Electrocochleography (ECoG), which objectively assesses cochlear potentials, is the indicated clinical procedure to analyze CMs. Although transtympanic ECoG yields recordings with higher amplitudes and lower test-retest variability, it has the disadvantage of being an invasive procedure. Extratympanic ECoG, therefore, is clinically more useful in this context, supporting an audiological diagnosis and increasing knowledge about cochlear function in AN/AD. ECoG is the most appropriate procedure for assessing cochlear function and helping identify CMs. CMs play an important role in the diagnosis of AN/AD. It is necessary and helpful to diagnose the sites of lesion in patients with AN/AD by analyzing the patterns of CM amplitudes. Therefore, recently ECoG has been used for diagnosing AN/AD. 8,23

Some patients with AN/AD lost their OAE over the period of time but there was no associated change in pure tone thresholds. It has also been reported that some patients with AN/AD do not have OAEs but rather evidence of hair cell function was evident from CM. Therefore these authors suggested that presence of $\mathrm{CM}$ with absent ABR seems to be reliable criteria for diagnosing AN/AD. ${ }^{24,25}$

There has been controversy regarding whether to provide hearing devices (hearing aids, personal radiofrequency [frequency modulation] systems, or cochlear implants) to children with AN/AD and whether to offer aural-oral or visual-manual modes of habilitation. ${ }^{14,26}$ Berlin et al suggested that conventional amplification has little beneficial effect on AN/AD patients. ${ }^{27}$ On the contrary, Cone-Wesson et al concluded that nearly $50 \%$ of children demonstrate some benefit from the use of conventional hearing aids and that a trial of amplification is warranted. ${ }^{14,21}$ Cochlear implantation may also be an option for hearing rehabilitation. Although the outcome of cochlear implantation in children with AN/AD might vary, it is favorable in most cases. Cochlear implantation seems a justified hearing rehabilitation option for children with AN/AD and limited benefits from conventional hearing aids. ${ }^{28-30}$ In our series, we obtained some development in hearing and speech abilities using a conventional hearing aid in one case.

The current position statement of The Joint Committee on Infant Hearing (2007) calls for (1) physiological hearing screening of all infants before they are 1 month old, (2) confirmation of the hearing loss before 3 months of age, and (3) commencement of an interdisciplinary intervention program before the infant is 6 months old. Moreover, the scope of disorders targeted for identification has been expanded to include neural hearing loss (especially AN/AD) in addition to sensorineural and permanent conductive hearing loss. ${ }^{31}$

\section{Conclusion}

Although AN/AD affects only a small portion of all persons with hearing loss, the infant, child, or adult with AN/AD is often most disabled by the hearing disorder because of the lack of knowledge about its cause and, more importantly, its treatment. Continued research regarding the causes and pathologies underlying this disorder is needed. ${ }^{10}$ However, it is also necessary to develop methods to reduce falsenegative screening results and to provide accurate diagnosis for the disorder. ${ }^{2} \mathrm{~A}$ combined OAE and ABR screening procedure may be considered to overcome the limitations of OAEonly procedures especially in high-risk infants. Also, it should be kept in mind that adult AN/D cases may be related with systemic neurological diseases.

\section{References}

1 Madden C, Rutter M, Hilbert L, Greinwald JH Jr, Choo DI. Clinical and audiological features in auditory neuropathy. Arch Otolaryngol Head Neck Surg 2002;128(9):1026-1030

2 Lee JSM, McPherson B, Yuen KCP, Wong LLN. Screening for auditory neuropathy in a school for hearing impaired children. Int J Pediatr Otorhinolaryngol 2001;61(1):39-46

3 Starr A, Picton TW, Sininger Y, Hood LJ, Berlin CI. Auditory neuropathy. Brain 1996;119(Pt 3):741-753 
4 Worthington DW, Peters JF. Quantifiable hearing and no ABR: paradox or error? Ear Hear 1980;1(5):281-285

5 Kraus N, Ozdamar O, Stein L, Reed N. Absent auditory brain stem response: peripheral hearing loss or brain stem dysfunction? Laryngoscope 1984;94(3):400-406

6 Doyle KJ, Sininger Y, Starr A. Auditory neuropathy in childhood. Laryngoscope 1998;108(9):1374-1377

7 Rapin I, Gravel J. "Auditory neuropathy": physiologic and pathologic evidence calls for more diagnostic specificity. Int J Pediatr Otorhinolaryngol 2003;67(7):707-728

8 Shi W, Ji F, Lan L, et al. Characteristics of cochlear microphonics in infants and young children with auditory neuropathy. Acta Otolaryngol 2012;132(2):188-196

9 Roush P, Frymark T, Venediktov R, Wang B. Audiologic management of auditory neuropathy spectrum disorder in children: a systematic review of the literature. Am J Audiol 2011;20(2):159-170

10 Nikolopoulos TP. Auditory dyssynchrony or auditory neuropathy: understanding the pathophysiology and exploring methods of treatment. Int J Pediatr Otorhinolaryngol 2014;78(2):171-173

11 Sinha SK, Barman A, Singh NK, Rajeshwari G, Sharanya R. Involvement of peripheral vestibular nerve in individuals with auditory neuropathy. Eur Arch Otorhinolaryngol 2013;270(8):2207-2214

12 Sawada S, Mori N, Mount RJ, Harrison RV. Differential vulnerability of inner and outer hair cell systems to chronic mild hypoxia and glutamate ototoxicity: insights into the cause of auditory neuropathy. J Otolaryngol 2001;30(2):106-114

13 Marco J, Morant A, Orts M, Pitarch MI, Garcia J. Auditory neuropathy in children. Acta Otolaryngol 2000;120(2):201-204

14 Cone-Wesson B, Rance G. Auditory neuropathy: a brief review. Curr Opin Otolaryngol Head Neck Surg 2000;8(5):421-425

15 Harrison RV. An animal model of auditory neuropathy. Ear Hear 1998;19(5):355-361

16 Salvi RJ, Wang J, Ding D, Stecker N, Arnold S. Auditory deprivation of the central auditory system resulting from selective inner hair cell loss: animal model of auditory neuropathy. Scand Audiol Suppl 1999;51(Suppl 51):1-12

17 Kraus N, Bradlow AR, Cheatham MA, et al. Consequences of neural asynchrony: a case of auditory neuropathy. J Assoc Res Otolaryngol 2000;1(1):33-45

18 Starr A, McPherson D, Patterson J, et al. Absence of both auditory evoked potentials and auditory percepts dependent on timing cues. Brain 1991;114(Pt 3):1157-1180
19 Kırkım G, Serbetçioğlu MB, Ceryan K. Auditory neuropathy in children: diagnostic criteria and audiological test results. Kulak Burun Bogaz Ihtis Derg 2005;15(1-2):1-8

20 Davis H, Hirsh SK. The audiometric utility of brain stem responses to low-frequency sounds. Audiology 1976;15(3):181-195

21 Rance G, Beer DE, Cone-Wesson B, et al. Clinical findings for a group of infants and young children with auditory neuropathy. Ear Hear 1999;20(3):238-252

22 Maris M, Venstermans C, Boudewyns AN. Auditory neuropathy/ dyssynchrony as a cause of failed neonatal hearing screening. Int J Pediatr Otorhinolaryngol 2011;75(7):973-975

23 Anastasio ART, Alvarenga KdeF, Costa Filho OA. Extratympanic electrocochleography in the diagnosis of auditory neuropathy/ auditory dyssynchrony. Braz J Otorhinolaryngol 2008;74(1): 132-136

24 Sanyelbhaa Talaat H, Khalil LH, Khafagy AH, Alkandari MM, Zein AM. Persistence of otoacoustic emissions in children with auditory neuropathy spectrum disorders. Int J Pediatr Otorhinolaryngol 2013;77(5):703-706

25 Mittal R, Ramesh AV, Panwar SS, Nilkanthan A, Nair S, Mehra PR. Auditory neuropathy spectrum disorder: its prevalence and audiological characteristics in an Indian tertiary care hospital. Int J Pediatr Otorhinolaryngol 2012;76(9):1351-1354

26 De Leenheer EM, Dhooge IJ, Veuillet E, Lina-Granade G, Truy E. Cochlear implantation in 3 adults with auditory neuropathy/ auditory dys-synchrony. B-ENT 2008;4(3):183-191

27 Berlin CI, Morlet T, Hood LJ. Auditory neuropathy/dyssynchrony its diagnosis and management. Pediatr Clin N Am 2003;50:331-340

28 Kontorinis G, Lloyd SK, Henderson L, et al. Cochlear implantation in children with auditory neuropathy spectrum disorders. Cochlear Implants Int 2014;15(Suppl 1):S51-S54

29 Budenz CL, Telian SA, Arnedt C, et al. Outcomes of cochlear implantation in children with isolated auditory neuropathy versus cochlear hearing loss. Otol Neurotol 2013;34(3):477-483

30 Cardon G, Sharma A. Central auditory maturation and behavioral outcome in children with auditory neuropathy spectrum disorder who use cochlear implants. Int J Audiol 2013;52(9): 577-586

31 American Academy of Pediatrics, Joint Committee on Infant Hearing. Year 2007 position statement: principles and guidelines for early hearing detection and intervention programs. Pediatrics 2007;120(4):898-921 